\title{
Triptolide downregulates Rac1 and the JAK/STAT3 pathway and inhibits colitis-related colon cancer progression
}

\author{
Zhipeng Wang ${ }^{1 *}$, Haifeng $\mathrm{Jin}^{2 *}$, Ruodan $\mathrm{Xu}^{3}$, \\ Qibing Mei ${ }^{1,4}$ and Daiming Fan ${ }^{2,4}$ \\ ${ }^{1}$ Department of Pharmacology \\ School of Pharmacy \\ ${ }^{2}$ State Key Laboratory of Cancer Biology and \\ Institute of Digestive Diseases \\ Xijing Hospital \\ The Fourth Military Medical University \\ Shaanxi Province 710032, China \\ ${ }^{3}$ Protein Laboratory \\ Department of Neuroscience and Pharmacology \\ University of Copenhagen \\ Copenhagen 2200, Denmark \\ ${ }^{4}$ Corresponding authors: qbmei@fmmu.edu.cn (Q. Mei), \\ fandmdmfan@yahoo.com.cn (D. Fan) \\ *These authors contributed equally to this work. \\ DOI 10.3858/emm.2009.41.10.078
}

Accepted 28 May 2009

Abbreviations: COX, cyclooxygenase; CRC, colorectal cancer; IBD, inflammatory bowel disease; JAK, Janus kinase; STAT, signal transducers and activators of transcription

\begin{abstract}
Triptolide, a diterpenoid triepoxide from the traditional Chinese medicinal herb Tripterygium wilfordii Hook. $\mathrm{f}$., is a potential treatment for autoimmune diseases as well a possible anti-tumor agent. It inhibits proliferation of coloretal cancer cells in vitro and in vivo. In this study, its ability to block progress of colitis to colon cancer, and its molecular mechanism of action are investigated. A mouse model for colitis-induced colorectal cancer was used to test the effect of triptolide on cancer progression. Treatment of mice with triptolide decreased the incidence of colon cancer formation, and increased survival rate. Moreover, triptolide decreased the incidence of tumors in nude mice inoculated with cultured colon cancer cells dose-dependently. In vitro, triptolide inhibited the proliferation, migration and colony formation of colon cancer cells. Secretion of IL6 and levels of JAK1, IL6R and phosphorylated STAT3 were all reduced by triptolide treatment. Triptolide prohibited Rac1 activity and blocked cyclin D1 and CDK4 expression, leading to G1
\end{abstract}

arrest. Triptolide interrupted the IL6R-JAK/STAT pathway that is crucial for cell proliferation, survival, and inflammation. This suggests that triptolide might be a candidate for prevention of colitis induced colon cancer because it reduces inflammation and prevents tumor formation and development.

Keywords: colonic neoplasms; interleukin-6; rac1 GTP-binding protein; STAT3 transcription factor; triptolide

\section{Introduction}

Traditional medicines represent an abundant source of potential pharmaceuticals. One of the most promising compounds from a traditional medical herb is triptolide, a diterpenoid triepoxide purified from the Thundergod vine, Tripterygium wilfordii Hook. f. Triptolide has anti-inflammatory effects, and has been the subject of clinical trials as a rheumatoid arthritis treatment (Corson and Crews, 2007; Qiu and Kao, 2003; Schmidt et al., 2007). Triptolide has a complex mechanism of action, suppressing production of prostaglandin E2 via down-regulation of cyclooxygenase (COX)-2, and reducing levels of cytokines, including, among others, IL-1 $\beta$, TNF and IL6 (Qiu and Kao, 2003; Lin et al., 2007). A known effect of triptolide is inhibition of the transcription factor NF-KB activation (Qiu and Kao, 2003; Wei et al., 2008), which can have manifold effects on inflammation and proliferation.

In addition to its anti-inflammatory action, several reports have indicated that triptolide can inhibit the proliferation of cancer cells in vitro and reduce the growth and metastases of tumors in vivo. Results with triptolide in vivo include inhibition of the growth of cholangiocarcinoma cells in hamsters (Tengchaisri et al., 1998), as well as of xenografts from human melanoma, breast cancer, bladder cancer, and gastric and coloral carcinoma lines in nude mice (Yang et al., 2003). Triptolide reduced the growth and spread of pancreatic tumors in mice (Phillips et al., 2007), and showed weak activity against solid tumors in a mouse breast cancer model (Shamon et al., 1997). It was also efficacious as a adjunct agent in combination chemotherapy against tumors developed from human ovarian cancer cells inoculated into nude mice (Westfall et al., 2008). In addition, clinical trials in China using 
triptolide have shown remission rates of $71 \%$ and $87 \%$ in mononucleocytic and granulocytic leukemia, respectively (Lu et al, 1992). Although the precise mechanism of its anti-cancer effects is unknown, triptolide has pro-apoptotic effects (Carter et al., 2006; Liang and Fu, 2008; Yao et al., 2008). triptolide ameliorates chronic colitis mediated by the acquired immune response in a mouse model (Wei et al., 2008), but no previous reports has examined the effect of triptolide on colon cancer induced by chronic colitis.

Inflammatory bowel disease (IBD), such as ulcerative colitis and Crohn's disease are associated with an increased risk for developing colorectal cancer and precancerous dysplastic epithelial changes (Krok and Lichtenstein, 2004). In the most recent model for progression from IBD to colorectal cancer (CRC), the pro-inflammatory cytokine IL6 has a key role as a mechanistic link between inflammation and colonic cancer (Lin and Karin, 2007, Atreya and Neurath, 2005b). Levels of both IL6 and a soluble form of the IL6 receptor (sIL6R), which is generated by limited proteolysis of the membrane-bound IL6 receptor, are elevated in IBD and CRC (Belluco et al., 2000; Mudter and Neurath, 2007). The signal generated by IL6 is transduced through Janus kinase (JAK) activation of Signal Transducers and Activators of Transcription (STAT), in particular STAT3. Aberrant regulation of STAT3regulated genes is associated with oncogenic transformation. Thus, all components of this pathway may play crucial roles in the pathogenic process that links intestinal inflammation and colonic cancer. In fact, high levels of IL6 and the soluble IL6 receptor (sIL6R) have been reported in several chronic inflammatory and autoimmune diseases as well as in cancer (Mudter and Neurath, 2007). In addition, IL6 is one part of an autocrine inflammatory loop involving Rac1, a small GTPase in the Rho family (Faruqi et al., 2001).

Specifically blocking IL6-regulated signalling pathways represents a promising approach for the therapy of IBD and colorectal cancer (Mudter and Neurath, 2007). In fact, antibodies against the sIL6 receptor effectively reduced inflammation in IBD and activated apoptosis (Atreya et al., 2001). With its anti-inflammatory and anti-cancer properties, triptolide is a natural candidate for treatment of IBD leading to colorectal cancer. We used a colitis-induced colon cancer mouse model to demonstrate that triptolide has an in vivo protective effect against IBD-induced cancer. Mechanistically, experiments with cultured colon cancer cell lines demonstrated that triptolide inhibits components of the IL6 and JAK/STAT3 signal transduction pathway, as well as through the small GTPase Rac1.

\section{Results}

\section{Triptolide reduces colitis-associated colorectal cancer and inhibits cultured cancer cells}

To evaluate directly whether triptolide prohibits incidence of colitis-associated colorectal cancer, we employed a DMH/DSS-induced mouse model for colitis-related colonic carcinogenesis. In DMH/DSS treated model mice, body weights slightly decreased. Three experimental groups were treated with triptolide at $0.1,0.3$ or $1 \mathrm{mg} / \mathrm{kg} /$ day for 20 weeks. No dramatic difference compared to control group was observed in body weights in mice treated by triptolide at the dose of $1 \mathrm{mg} / \mathrm{kg} /$ day. Survival rates were investigated, and $54.4 \%$ mice in DMH/DSS model group survived the treatment period. Meanwhile, administration of triptolide increased survival rates dose-dependently, up to $100 \%$ in $1 \mathrm{mg} / \mathrm{kg}$ dosage group. In the DMH/DSS-induced model group, the incidence of tumors was $100 \%$, whereas no tumors were observed in the corresponding control mice treated with saline. The incidences of colorectal tumors in triptolide-treated groups were significantly lower (8 out of 10,6 out of 10 and 3 out of 9 ) than that of the control group (Table 1).

Table 1. Triptolide prevents mice from colitis related colon tumor formation.

\begin{tabular}{|c|c|c|c|c|c|c|}
\hline Group & $\mathrm{n}$ & $\begin{array}{c}\text { Body } \\
\text { Weight }(\mathrm{g})\end{array}$ & $\begin{array}{l}\text { Survival } \\
\text { rate }(\%)\end{array}$ & $\begin{array}{c}\text { Incidence of } \\
\text { mucosal } \\
\text { ulcer (\%) }\end{array}$ & $\begin{array}{l}\text { Incidence } \\
\text { of Tumor }\end{array}$ & $\begin{array}{l}\text { Number } \\
\text { of Tumors }\end{array}$ \\
\hline Control & 8 & $45.1 \pm 2.9$ & 100 & 0 & 0 & 0 \\
\hline DMH/DSS treated & 9 & $42.2 \pm 2.7$ & 54.4 & 100 & 9 & 13 \\
\hline $\begin{array}{l}\text { Triptolide treated }(0.1 \mathrm{mg} / \mathrm{kg}) \\
\text { +DMH/DSS }\end{array}$ & 10 & $42.5 \pm 3.1$ & 60 & 100 & 8 & 11 \\
\hline $\begin{array}{l}\text { Triptolide treated }(0.3 \mathrm{mg} / \mathrm{kg}) \\
\text { +DMH/DSS }\end{array}$ & 10 & $43.9 \pm 5.2$ & 80 & 90 & 6 & 8 \\
\hline $\begin{array}{l}\text { Triptolide treated }(1 \mathrm{mg} / \mathrm{kg}) \\
+ \text { +DMH/DSS }\end{array}$ & 9 & $44.6 \pm 3.9$ & 100 & 66.7 & 3 & 3 \\
\hline
\end{tabular}


Subsequently, the occurrence of colon tumors was significantly lower in triptolide-treated groups than in DMH/DSS treated mice. Moreover, colonic mucosal ulcerations were found in the distal colon of all of mice in model group. Nevertheless, in triptolide treated group $(1 \mathrm{mg} / \mathrm{kg})$, incidence of mucosal ulcer dropped to $66.7 \%$.

Having confirmed the hypothesis that triptolide has an in vivo effect on colitis-induced colon cancer, we investigated the molecular and cellular effects of triptolide using two human colorectal carcinoma cell lines, SW480 and Caco 2. Focus formation in the two lines was dramatically reduced by treatment with $100 \mathrm{nM}$ triptolide. Untreated SW480 cells and Caco 2 cells, plated at 500 cells per $60 \mathrm{~mm}$ plates, formed $98 \pm 19$ and $95 \pm 21$ colonies, respectively, while the same number of cells treated with $100 \mathrm{nM}$ triptolide formed $63 \pm 18$ and $55 \pm 16$ colonies. Cell migration was investigated using a monolayer wound healing assay. Quantification of cell movement over $72 \mathrm{~h}$ showed migration was inhibited by $25 \pm 11 \%$ and $30 \pm 9 \%$ in SW480 and Caco 2 cells by $100 \mathrm{nM}$ triptolide (Figure 1A and 1B). We next investigated proliferation of the colorectal cancer cells after triptolide treatment. MTT reduction results showed that triptolide significantly decreased SW480 and Caco 2 cell proliferation in dose-dependent manner (Figure 2A and 2B).

Evidence has accumulated for the participation of Rho GTPases, including RhoA, Cdc42 and Rac1, in cell proliferation, transformation, migration, invasion and metastasis. We therefore evaluated the activity of Rho GTPases by pull down assay, after cells were treated by triptolide at the concentrations of 0,30 or $100 \mathrm{nM}$ for $24 \mathrm{~h}$. Although Rac1

\section{A}
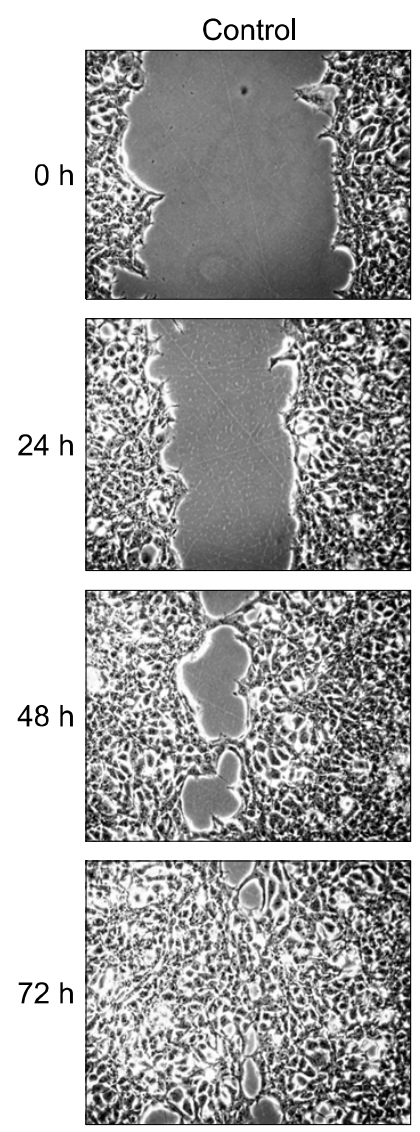
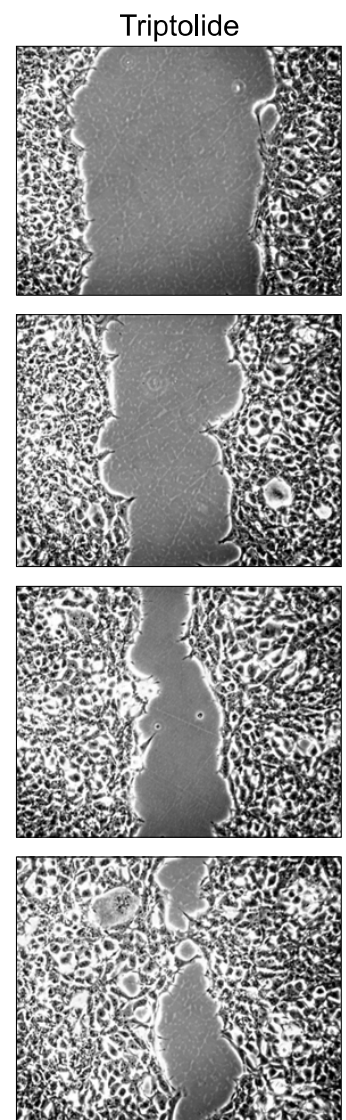

B

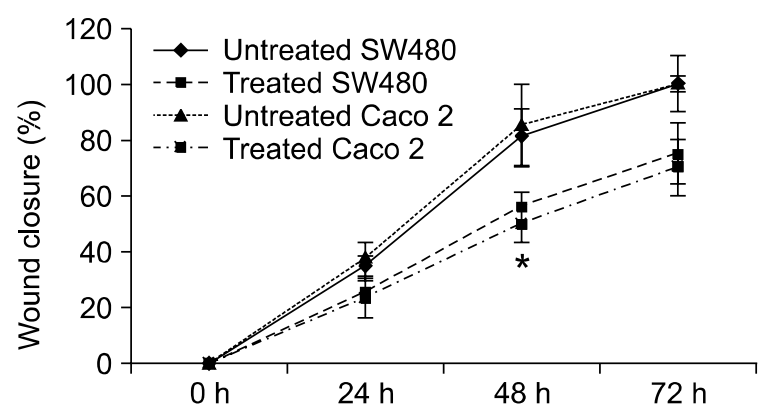

C

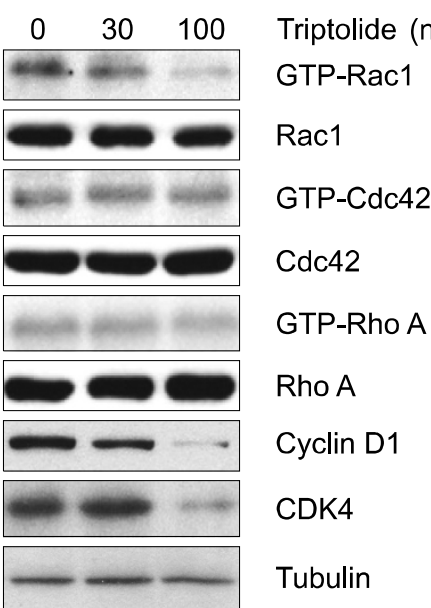

Figure 1. Triptolide inhibits colorectal cells migration. (A) Wound healing assay of colon cancer cell lines SW480 and Caco 2 cells. Wound (1 mm wide) was performed after confluence, and cells were treated by triptolide at 0 or $100 \mathrm{nM}$. Migration was assessed every $24 \mathrm{~h}$. (B) Quantification of wound closure. Graphic represents the wound width as the mean s.e.m. of the \% of the closure of original wound in triplicate plates. Similar results were obtained in three experiments. ${ }^{*} P<0.05$ comparing to the same time points of wound width of control SW480 or Caco 2 cells. Student's $t$-test was used to evaluate statistically significant differences between the values. (C) Effect of triptolide on RhoGTPases Rac1, Cdc42, RhoA, Cyclin D1 and CDK4. Cells were exposed to triptolide at $0,30,100 \mathrm{nM}$ for $24 \mathrm{~h}$. Cell lysates were made. Expression of Rac1, Cdc42, RhoA, Cyclin D1 and CDK4 were analyzed by Western blots. Activities of Rac1, Cdc42 and RhoA were analyzed by pull down assay. 

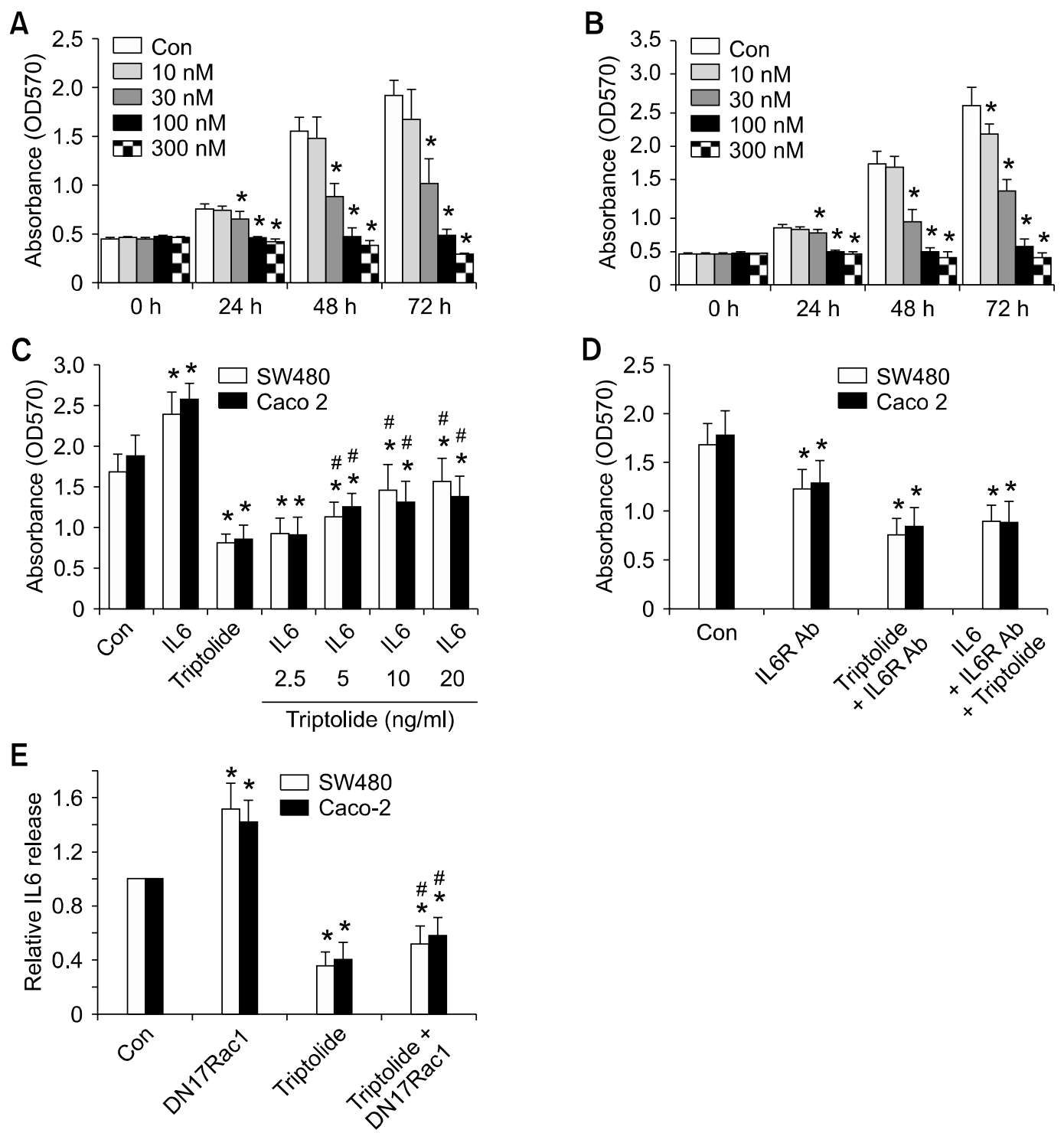

$\mathbf{F}$

Parental

Empty vector

DN17-Rac1

Triptolide
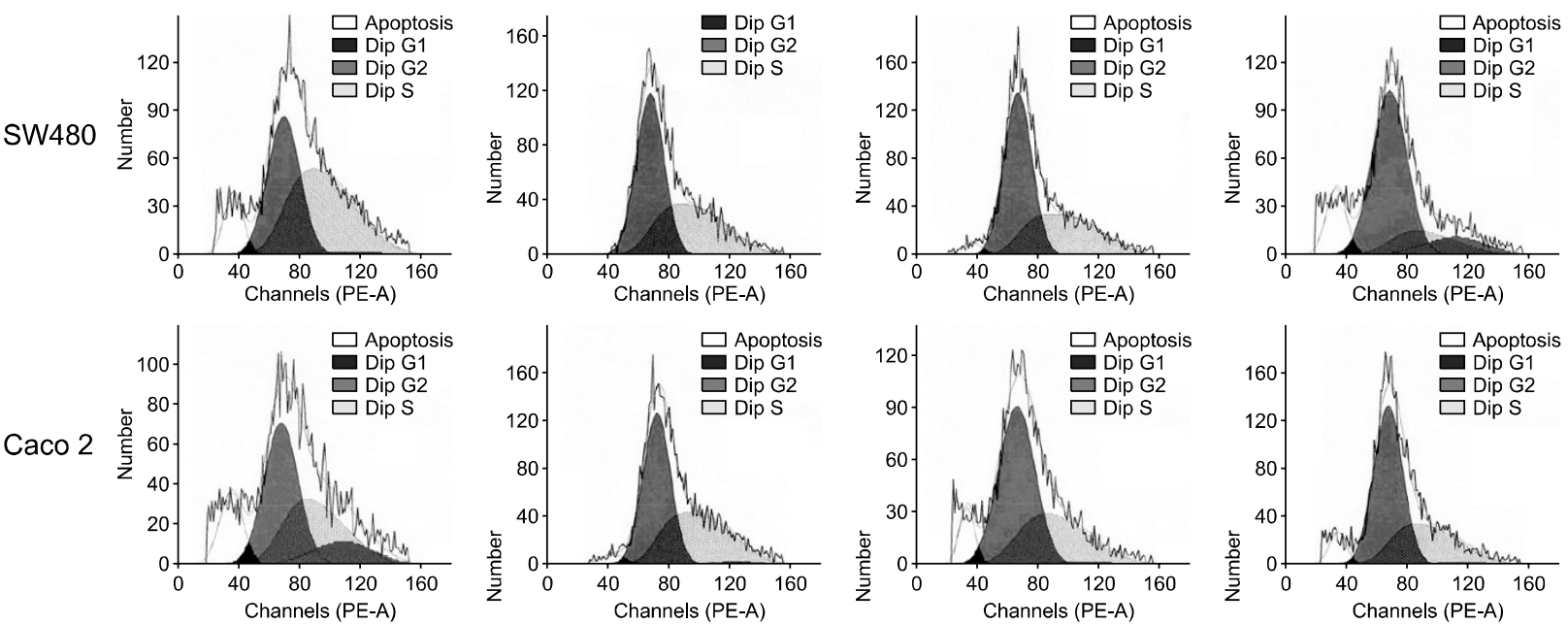
Figure 2. Triptolide inhibits proliferation in colon cancer cell lines SW480 and Caco 2. (A, B) Dose-dependent effects of proliferation assays in SW480 and Caco 2 cells treated by triptolide at 10,30,100, $300 \mathrm{nM}$ were measured by MTT assay. Samples from a representative experiment that was repeated three times. ${ }^{*} P<0.05$ comparing to untreated control cell lines of SW480 or Caco 2. Student's $t$-test was used to evaluate statistically significant differences between the values. (C) SW480 and Caco 2 cells were exposed to triptolide (100 nM), recombinant human IL6 (2.5, 5, 10 or 20 $\mathrm{ng} / \mathrm{ml}$ ) and triptolide plus IL6 on proliferation for $24 \mathrm{~h}$, proliferation was measured by MTT assay. Similar results were obtained in three experiments. ${ }^{*} P$

0.05 comparing to untreated con cell lines of SW480 or Caco $2 .{ }^{\#} P<0.05$ comparing to triptolide treated con cell lines of SW480 or Caco 2. Student's $t$-test was used to evaluate statistically significant differences between the values. (D) SW480 and Caco 2 cells were exposed to triptolide (100 $\mathrm{nM})$, neutrolising IL6R antibody $(20 \mu \mathrm{g} / \mathrm{ml})$, triptolide or triptolide plus IL6 $(20 \mathrm{ng} / \mathrm{ml})$ on proliferation for $24 \mathrm{~h}$, proliferation was measured by MTT assay. Similar results were obtained in three experiments. ${ }^{*} P<0.05$ comparing to untreated control cell lines of SW480 or Caco 2. Student's $t$-test was used to evaluate statistically significant differences between the values. (E) Effect of DN Rac1 and triptolide on secretion of IL6 in SW480 and Caco 2 cells were measured by ELISA assay. Similar results were obtained in three experiments. ${ }^{*} P<0.05$ comparing to untreated control cell lines of SW480 or Caco 2. ${ }^{\#} P<0.05$ comparing to triptolide treated con cell lines of SW480 or Caco 2. Student's $t$-test was used to evaluate statistically significant differences between the values. (F) SW480 and Caco 2 cells were treated by triptolide (100 nM) or DN17-Rac1, cell cycle progression was analyzed by FACs. These figures were representatives of three independent experiments with identical results.

expression levels were unaffected by increasing concentrations of triptolide, Rac1 activity decreased, whereas the activity of RhoA and Cdc42 were not obviously changed (Figure 1C). Cell-cycle analysis was performed and revealed that $\mathrm{G} 1$ phase cells accumulated rapidly after triptolide treatment, indicating G1/S arrest (Figure 2F). Cyclin D1 is one of the essential cyclins, regulated by Rac1, that regulate G1-to S-phase transition during cell-cycle progression. Western blot results indicated repression of CyclinD1 and CDK4 by triptolide treatment in a dose-dependent manner (Figure 1C).

Since IL6 is thought to stimulate progression of IBD-induced colorectal cancer, we tested whether IL6 would affect the inhibition of proliferation in the SW480 and Caco 2 cell lines. Triptolide and IL6 had antagonistic effects and incubation of triptolide-treated cells with $2.5,5,10,20 \mathrm{ng} / \mathrm{ml}$ recombinant human IL6 partially overcame triptolide inhibition in a dose-dependent manner (Figure 2C). Moreover, inhibition of IL6 function by neutralizing IL6R antibody $(20 \mu \mathrm{g} / \mathrm{ml})$ (Chugai Pharmaceuticals, Shizuoka, Japan) prohibited cells proliferation. Incubation of triptolide with IL6R antibody blocked proliferation significantly, meanwhile, treatment of IL6 $(20 \mathrm{ng} / \mathrm{ml})$ could not rescue this inhibition. This suggested that triptolide affects the signal transduction pathway stimulated by IL6.

\section{Triptolide inhibits IL6R and JAK1 expression, and STAT3 phosphorylation}

In its pathogenic role in IBD and colorectal cancer, IL6 is thought to act through the JAK/STAT3 signaling pathway (Atreya and Neurath, 2005b). We therefore evaluated the effects of triptolide on the IL6-STAT3 pathway. Cells were exposed to 0 , 30 or $100 \mathrm{nM}$ triptolide for $24 \mathrm{~h}$. While the levels of STAT3 protein remained constant, serving as a control for general protein expression, phosphorylation of STAT3, as well as levels of IL6 receptor
(IL6R) and JAK1, decreased after triptolide treatment (Figure 3). This suggested that triptolide might affect colitis and colon cancer formation through inhibition of the IL6-JAK/STAT pathway.

\section{Triptolide affects IL6-JAK/STAT via Rac1}

Rac1 has been implicated in the regulation of STAT3 transcriptional activity, either indirectly (Faruqi et al., 2001), or through direct binding to STAT3 that promotes activating phosphorylation and nuclear localization (Simon et al., 2000; Simeone-Penney et al., 2008). Given the effect of triptolide on STAT3 phosphorylation and Rac1 activation, and the functional connection between Rac1 and STAT3, we examined cross-talk between Rac1 and the JAK/STAT pathway. Inhibition of Rac1 activity by transformation with a dominant negative (DN17-Rac1) did not change levels of IL6R, JAK1 or STAT3 proteins, but STAT3 phosphorylation appeared to increase relative to a control cells transformed with empty vector (Figure 4A). Cell cycle analysis had shown G1 arrest (Figure 2F) after triptolide treatment and in fact, cells with the DN17-Rac1 showed decreased levels of CyclinD1 and CDK4 (Figure 4A). Downregulation of these cell-cyle regulators may explain $\mathrm{G} 1$ arrest after triptolide treatment. On contrary, transformation of constitutively activated Rac1 (CA-Rac1) resulted in decrease of phospho STAT3, as well as elevation of CyclinD1 and CDK4. Finally, immunoprecipitation demonstrated that, consistent with previous studies (Simon et al., 2000; SimeonePenney et al., 2008), Rac1 bound STAT3 directly, while no direct interaction was detected between Rac1 and JAK1 or IL6R (Figure 4B).

Rac1 has been shown to affect IL6 expression and secretion in smooth muscle cells and in cultured cells (Faruqi et al., 2001; Zampetaki et al., 2005). We found that incubation of colon cancer cell lines with triptolide decreased active form of 
Rac1, GTP-bound Rac1, in a dose-dependent manner (Figure 1C) and also markedly inhibited the secretion of IL6 (Figure 2D). Although DN Rac1 promoted activity of STAT3, which could increase IL6 production, triptolide dramatically inhibited IL6R expression, which subsequently eliminated IL6 production. As a consequence, incubation of triptolide inhibited the secretion of IL6 (Figure 2D).

\section{Triptolide treatment prohibits growth of primary tumors in nude mice}

We returned to the in vivo analysis of triptolide on colon cancer with a better understanding of its molecular effects. When triptolide was administered to mice with established primary tumor xenografts, the results were dramatic. Figure $5 \mathrm{~A}$ showed that triptolide had a significant inhibitory effect on the growth of all tumor cell lines in a
A

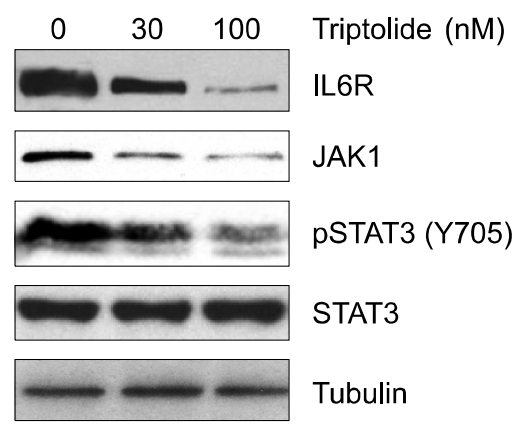

B
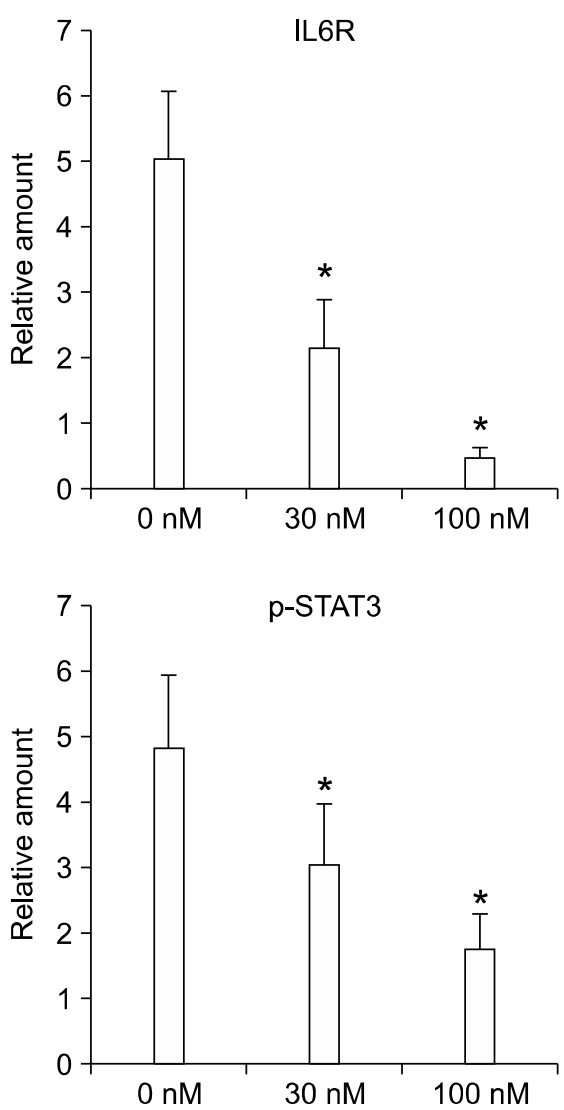
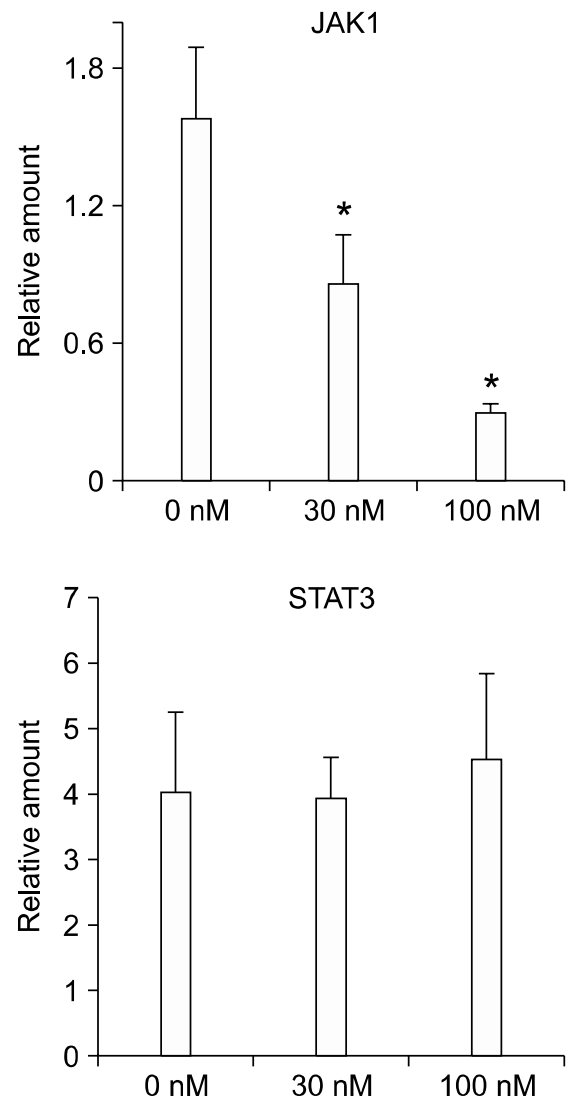

Figure 3 Triptolide effects on IL6R/JAK1/STAT3 pathway. SW480 cells were treated by triptolide at $100 \mathrm{nM}$ for $24 \mathrm{~h}$. (A) Cell lysates were prepared. JAK1 and IL6R expression, and STAT3 phosphorylation were detected by western blot with antibodies against IL6R, JAK1, STAT3 and phosphorylated STAT3. (B) Graphs are represented as the mean density of IL6R, JAK1, p STAT3 and STAT3 bands normalised against the mean density of tubulin band from three independent experiments (presented as relative density of individual protein). ${ }^{*} P<$ 0.05 comparing to untreated control cell lines of SW480. Student's $t$-test was used to evaluate statistically significant differences between the values. 
dose-dependently. Western blots of protein extracts from the tumor xenografts showed that triptolide had the same effects in vivo as in vitro. Levels of IL6R and JAK1, and phosphorylation of STAT3 were inhibited in the tumors of mice treated with triptolide. Although Rac1 levels were unchanged, Pull down results showed active Rac1 was significantly inhibited (Figure 5B). Furthermore, growth of DN17-Rac1 transformed cells was markedly lower than non-transformed tumor cells in nude mice, nevertheless, CA-Rac1 transformed cell grew faster than non-transformed tumor cells (Figure 5C).

\section{Discussion}

Although anti-inflammatory agents like steroids are effective at reducing symptoms of IBD like Crohn's disease, new treatments that do not induce dependency are needed. In addition, treating the inflammation of IBD is not necessarily inhibit the progression to $\mathrm{CRC}$, so ideally, therapies would address both aspects of IBD (Atreya and Neurath, 2005a). Monoclonal antibodies against sIL6R show promise as anti-IBD and potentially anti-cancer agents (Atreya et al., 2001), but a less expensive alternative would be useful. Here we show that triptolide, which can be extracted from a Chinese medicinal plant, acts both in vitro and in vivo to inhibit CRC tumor formation. Both its anti-inflammatory and its anti-cancer activity are confirmed in a mouse model for CRC induced by inflammation.
In SW40 and Caco 2 colon carcinoma cell lines, triptolide inhibited cell migration and cell proliferation, causing an arrest in G1. Triptolide inactivated Rac1, a small GTPase in the Rho family that has clear roles in carcinogenesis. Rac1 is implicated in regulation of cell cycle regulators (Simeone-Penney et al., 2008), and we found that inhibition of Rac1 activation with a dominant negative allele reduced levels of CyclinD1 and CDK4. Thus, even if the evidence is indirect, a possible hypothesis for the G1 arrest seen when the colon carcinoma lines were treated with triptolide, is that active Rac1 was suppressed, leading to lower levels of cell cycle target genes. Since the Rho GTPases also act in the migration and invasion of cells (Ridley, 2001), reduction of Rac1 activity by triptolide results in the cell migration and focus formation deficiencies that we observed.

Triptolide also had significant effects on IL6-JAK/ STAT3 pathway that is crucial for cell proliferation, survival, and the inflammation reaction. It is thought to be a mechanism by which IBD progresses to CRC (Atreya and Neurath, 2005b). Triptolide treatment of colon carcinoma cells reduced IL6 and JAK1 levels, and although STAT3 levels remained constant, STAT3 phosphorylation decreased. Rac1 has been shown to stimulate expression of STAT3, IL6 and the IL6 receptor (Faruqi et al., 2001). Consistent with this study, our results point to active Rac1 increasing IL6 production. In contrast to Faruqi et al., but similar to other previous reports (Simon et al., 2000; Simeone-Penney et al., 2008),
A

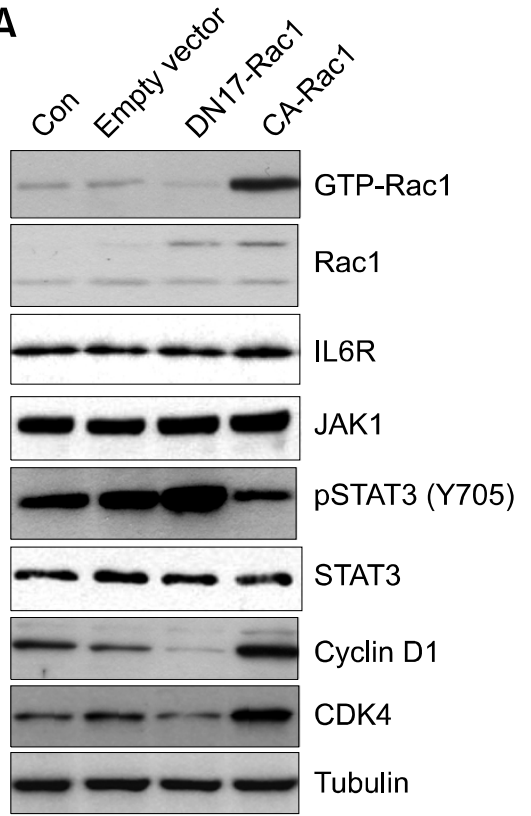

B

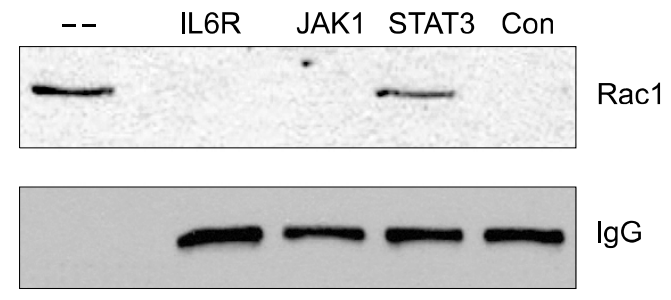

Figure 4. Rac1 inhibits JAK1/STAT3 pathway by binding to STAT3. (A) Western blot for the indicated proteins was performed in untransformed SW480 cells, SW480 cells transformed with empty vector, DN17Rac1 and CA-Rac1. (B) Immunoprecipitation of Rac1 and IL6R, JAK1 and STAT3. Immunoprecipitation was carried out with the antibody indicated on the top of the figure, followed by western blot for Rac1 or $\lg \mathrm{G}$ as indicated. 
we found that Rac1 bound directly to STAT3 and affected its phosphorylation state. However, in contrast to results from Simon et al. (2000) in which STAT3 phosphorylation decreased with transformation of a DN Rac1, we found that in the colorectal cell lines, STAT3 phosphorylation increased with DN Rac1 and decreased with CA Rac1. This could be because the Simon et al. (2000) study induced STAT3 phosphorylation by treating immortalized but non-cancerous COS-1 cells with epidermal growth factor, while we examined the steady-state level of STAT3 phosphorylation in cultured cells of cancerous origin. In any cases, we found that triptolide clearly reduced both the active form of Rac1 and STAT3 phosphorylation. Althourgh our results with DN Rac1 suggested that suppressing Rac1 may promote IL6 release, thereby increasing inflammation and STAT3 activation, triptolide decreased IL6R expression and consequently strongly reduced IL6 release, even in the presence of DN Rac1. Its profound effect on cancer cell proliferation was only partially affected by addition of recombinant IL6. This rescue could not happen after cells were treated with IL6R antibody and triptolide, indicating that IL6-JAK/ STAT3 pathway is a crucial pathway affected by triptolide.

In vivo, inflammation leading to colorectal tumors was induced by the genotoxic carcinogen $\mathrm{DMH}$ in mice. Triptolide significantly reduced colonic mucosal ulcerations and tumor growth in this colitis-induced colon cancer model. It was also effective against primary tumors of SW480 and Caco 2 xenografts in nude mice, showing the same mechanism of action that was seen for these cells in vitro, that is, reduction of GTP-Rac1, IL6R, JAK1
A

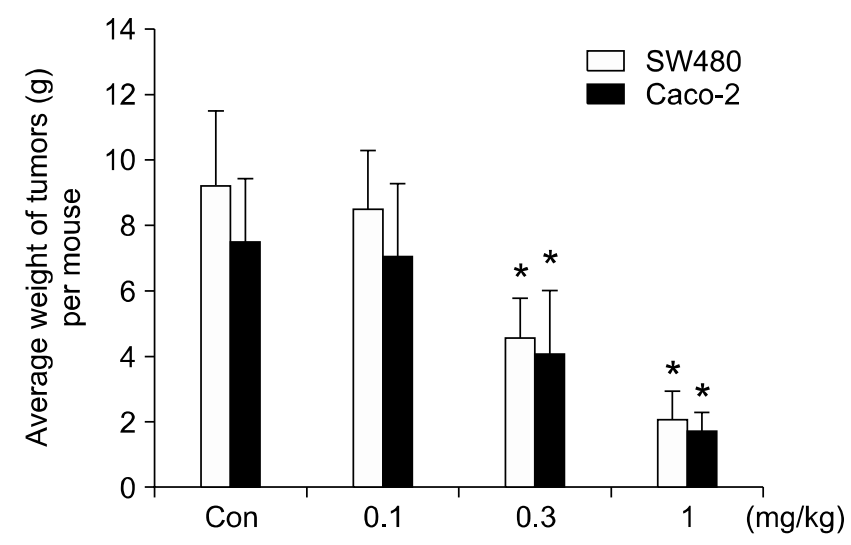

B

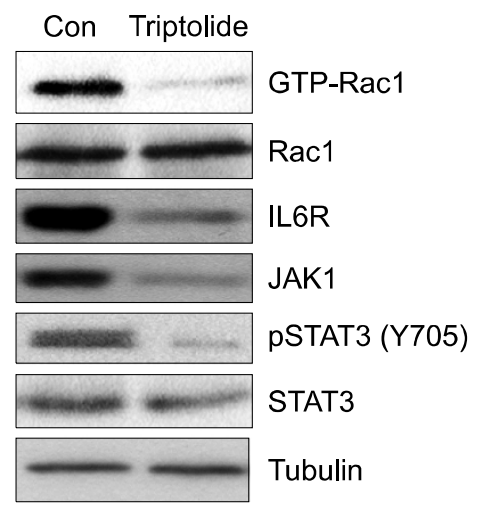

C

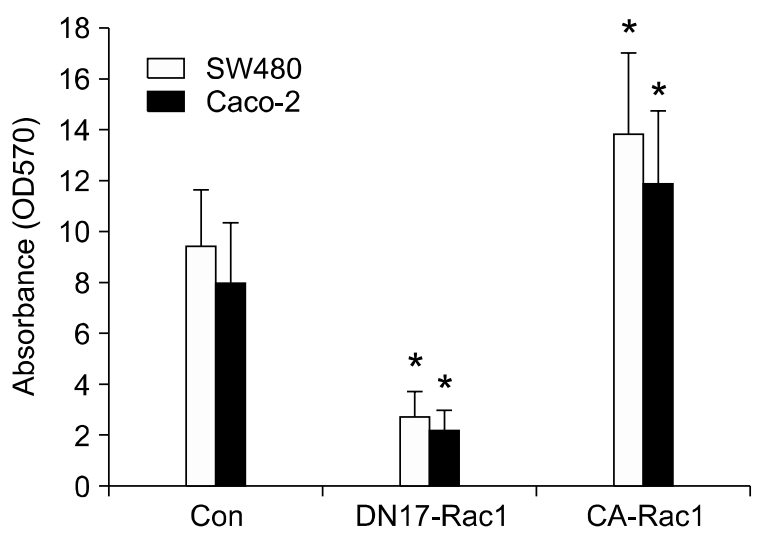

Figure 5. Tripolide inhibits tumor formation in nude mice. (A) SW480 and Caco 2 cells were injected subcutaneously into nude mice. Triptolide at $0,0.1$, 0.3 or $1 \mathrm{mg} / \mathrm{kg} /$ day was orally administrated daily for three weeks, and tumor xenografts were removed and weighed. ${ }^{*} P<0.05$ comparing to untreated mice. (B) Protein lysates were prepared from tumors. Rac1, IL6R, JAK1, STAT3 and pSTAT3 were detected by western blot. Rac1 activity was analyzed by pull down assay. (C) DN17-Rac1 and CA-Rac1 transformed SW480 and Caco 2 cells were injected subcutaneously into nude mice. Tumor xenografts were removed and weighed after three weeks. ${ }^{*} P<0.05$ comparing to tumors from untransformed cell lines of SW480 or Caco 2. 
and phosphorylation of STAT3. Growth of primary tumors from DN17-Rac1 transformed cells significantly slower than non-transformed cells in nude mice. On the contrary, growth of tumors from CA-Rac1 transformed cells was quicker, compared to those from non-transformed cells. These suggested Rac1 played an essential role in tumor growth. Taken together, triptolide blocked proliferation of tumor cells in vivo by interference of IL6-JAK/STAT3 and inactivation of Rac1.

Some limitations to the use of triptolide in human include its toxicity, its anti-fertility activity and the potential complexity of Tripterygium extracts (Brinker et al., 2007). One of its mechanisms of action is to trigger apoptosis, although a common problem with anti-cancer drugs is induction of programmed cell death in both normal and tumorous cells (Carter et al., 2006; Yao et al., 2008). Nonetheless, triptolide has potential not only as an anti-inflammatory, but, in its capacity as a downregulator of the JAK/STAT3 pathway, as an anti-cancer compound. This combination makes it especially suitable for cancers induced by chronic inflammation like ulceritis-induced colorectal cancer, as it could lessen inflammatory symptoms, while reducing the risk for progression to cancer.

\section{Methods}

\section{Chemicals}

Triptolide (Molecular Weight $=360.4$, purity $>99 \%$ ) was purchased from Sinobest Inc (Tongling, Anhui, China). It is a diterpenoid triepoxide, and its chemical structure is shown in Supplemental data Figure S1. 1,2-dimethyl hydrazine (DMH), dextran sodium sulfate (DSS), propidium iodide (PI) and MTT were supplied by Sigma-Aldrich (St. Louis, MO). G418 was obtainded from GibcoBRL (Bethesda, MD)

\section{Animals}

As an animal model for colitis-related carcinogenesis described previously (Onose et al., 2003), male Crj: CD-1 (ICR) mice were given a single intraperitoneal administration of $15 \mathrm{mg} / \mathrm{kg}$ body weight of the genotoxic colonic carcinogen, $\mathrm{DMH}$. This was followed by one week of oral exposure to a non-genotoxic carcinogen, DSS, at $2 \%$ in drinking water. At week 20 , mice were sacrificed. Mice were divided into five groups, normal group, model group, and triptolide treated groups $(0.1,0.3,1 \mathrm{mg} / \mathrm{kg} / \mathrm{day})$. It was dissolved with $0.9 \%$ saline and orally administered at 0.1 , $0.3,1 \mathrm{mg} / \mathrm{kg} / \mathrm{day}$ for 20 weeks. At the termination of the study, colons in mice were carefully removed and inspected for macroscopic pathological lesions.

To establish xenografts of colorectal cancer cells in mice, cells were injected subcutaneously into the flanks of 5 to 6 -week-old $\mathrm{BABL} / \mathrm{c}$ nude mice, at $5 \times 10^{6} \mathrm{cells} / \mathrm{site}$, in 8 mice/group. After growing for 3 days, the tumor xenografts reached approximately $100 \mathrm{~mm}^{3}$. Thereafter, triptolide at 0 , $0.1,0.3$ or $1 \mathrm{mg} / \mathrm{kg} /$ day was orally administrated on a daily basis. At the end of three weeks, mice were sacrificed, and tumor xenografts were removed and weighed.

\section{Cell culturing and assays}

SW480 and Caco 2 cells were cultured in RPMI 1640 supplemented with $10 \%$ heat-inactivated FBS, $100 \mathrm{U} / \mathrm{ml}$ penicillin, $100 \mathrm{mg} / \mathrm{ml}$ streptomycin, and $2 \mathrm{mM}$ glutamine. Cells were incubated at $37^{\circ} \mathrm{C}$ in a humidified atmosphere $\left(\begin{array}{lll}5 \% & \mathrm{CO}_{2}\end{array}\right)$. Treatment with recombinant human IL6 (Upstate Biotechnology, Lake Placid, NY) was at $6 \mathrm{ng} / \mathrm{ml}$, for $48 \mathrm{~h}$. Transfections of cells were carried out as described previously (Jefferies et al., 2000) using $5 \mu \mathrm{g} / \mathrm{ml}$ DN17-Rac1 plasmid, CA-Rac1, or empty plasmid. G418 selection at $200 \mu \mathrm{g} / \mathrm{ml}$ was applied $24 \mathrm{~h}$ after transfection.

Cell viability was determined by MTT assay. Briefly, cells were seeded at an initial density of $5 \times 10^{4}$ cells $/ \mathrm{ml}$ in a 96-well plate for $24 \mathrm{~h}$. Cells were then incubated with fresh medium containing various triptolide at $0,10,30,100$ or $300 \mathrm{nM}$ for $24 \mathrm{~h}, 48 \mathrm{~h}$ or $72 \mathrm{~h}$. After incubation, MTT was added into each well at a final concentration of $0.5 \mathrm{mg} / \mathrm{ml}$. The insoluble formazan was collected, dissolved in dimethylsulfoxide and measured with an ELISA reader (Bio-Rad) at a wavelength of $570 \mathrm{~nm}$.

For monolayer wound-healing assays, a total of $2 \times 10^{5}$ cells were collected and plated in a 12-well plate. At $100 \%$ confluence, two parallel wounds of $1 \mathrm{~mm}$ were made using a pipette tip. Wound size after $0,24,48$ and $72 \mathrm{~h}$ was measured using Zeiss LSM Image Browser software, version 3.1, in three independent experiments.

For focus formation assays, 500 cells were plated into $60 \mathrm{~mm}$ dishes for overnight growth, after which triptolide was added to a final concentration of $100 \mathrm{nM}$. After 14 days, colonies were washed twice with PBS, stained with $1 \%$ crystal violet, and counted on crystal-violet-stained dishes. All experiments were independently repeated at least three times.

Cell cycle analysis was performed by suspending cells in hypotonic solution $(0.1 \%$ Triton X-100, $1 \mathrm{mM}$ Tris- $\mathrm{HCl}$ $\mathrm{pH}$ 8.0, $3.4 \mathrm{mM}$ sodium citrate, $0.1 \mathrm{mM}$ EDTA) and staining with $50 \mu \mathrm{g} / \mathrm{ml}$ of $\mathrm{Pl}$. The population of cells in each cell-cycle phase was determined using ModiFIT software (Becton Dickinson).

\section{Cytokine assays, Western blots and immunoprecipitation assays}

Cells were grown in $25 \mathrm{~cm}^{2}$ flask in standard growth medium until $80-90 \%$ confluence was attained. Cells were washed in serum-free medium three times and incubated in the same medium for $24 \mathrm{~h}$. The medium was collected by centrifugation, and protein content in the medium was estimated using a commercial protein assay kit with BSA standards according to the manufacturer's instructions (Pierce, Rockford, IL). The IL-6 sandwich ELISA was performed using a sandwich ELISA kit (Biosource International, Camarillo, CA) according to the manufacturer's instruction. All data were normalised for total protein and expressed as $\mathrm{pg} \mathrm{mg}^{-1}$ of protein. 
Western blot and immunprecipitation assays were done as described previously (Jefferies et al., 2000). Bands were scanned and quantified with the programme QuantityOne (BioRad, Munich, Germany). Anti-Rac1, anti-Cdc42, and anti-RhoA antibodies were obtained from Transduction Laboratories. Anti-IL6R, anti-JAK1, anti-STAT3, anti-phospho-STAT3 (Y750), anti-CyclinD1 and anti-CDK4 were purchased from Santa Cruz Biotechnology (Santa Cruz, $\mathrm{CA})$.

\section{Rho GTPases activity assay (pull down)}

Rho activities were determined as described previously (Malliri et al. 2004). GTP-bound, active Rac (Rac-GTP) was precipitated by bounding to glutathione-coupled $\mathrm{Se}$ pharose beads. GTP-Rac1, GTP-RhoA or GTP-Cdc42 were detected by immunoblotting with anti-Rac1, anti-RhoA or anti-Cdc42 antibody.

\section{Supplemental data}

Supplemental Data include a figure and can be found with this article online at http://e-emm.or.kr/article/article_files/ SP-41-10-04.pdf.

\section{Refrences}

Atreya R, Mudter J, Finotto S, Mullberg J, Jostock T, Wirtz S, Schutz M, Bartsch B, Holtmann M, Becker C. Blockade of interleukin 6 trans signaling suppresses T-cell resistance against apoptosis in chronic intestinal inflammation: evidence in crohn disease and experimental colitis in vivo. Nat Med 2001;6:583-8

Atreya R, Neurath MF. Involvement of IL-6 in the pathogenesis of inflammatory bowel disease and colon cancer. Clin Rev Allergy Immunol 2005a;28:187-96

Atreya R, Neurath MF. Signaling molecules: the pathogenic role of the IL-6/STAT-3 trans signaling pathway in intestinal inflammation and in colonic cancer. Curr Drug Targets 2005b; 9:369-74

Belluco C, Nitti D, Frantz M, Toppan P, Basso D, Plebani M, Lise M, Jessup JM. Interleukin-6 blood level is associated with circulating carcinoembryonic antigen and prognosis in patients with colorectal cancer. Ann Surg Oncol 2000;7: 133-8

Brinker AM, Ma J, Lipsky PE, Raskin I. Medicinal chemistry and pharmacology of genus Tripterygium (Celastraceae). Phytochemistry 2007;68:732-66

Carter BZ, Mak DH, Schober WD, McQueen T, Harris D, Estrov Z, Evans RL, Andreeff M. Triptolide induces caspasedependent cell death mediated via the mitochondrial pathway in leukemic cells. Blood 2006;108:630-37

Corson TW, Crews CM. Molecular understanding and modern application of traditional medicines: triumphs and trials. Cell 2007;130:769-74

Faruqi TR, Gomez D, Bustelo XR, Bar-Sagi D, Reich NC. Rac1 mediates STAT3 activation by autocrine IL-6. Proc Natl

\section{Acad Sci U S A 2001;98:9014-19}

Jefferies CA, O'Neill LAJ. Rac1 regulates interleukin 1-induced nuclear factor $\mathrm{kB}$ activation in an inhibitory protein $\mathrm{kBa}$-independent manner by enhancing the ability of the p65 subunit to transactivate gene expression. J Biol Chem 2000; 275:3114-20

Krok KL, Lichtenstein GR. Colorectal cancer in inflammatory bowel disease. Curr Opin Gastroenterol 2004;20:43-8

Liang M, Fu J. Triptolide inhibits interferon-gamma-induced programmed death-1-ligand 1 surface expression in breast cancer cells. Cancer Lett 2008;270:337-41

Lin N, Liu C, Xiao C, Jia H, Imada K, Wu H, Ito A. Triptolide, a diterpenoid triepoxide, suppresses inflammation and cartilage destruction in collagen-induced arthritis mice. Biochem Pharmacol 2007;73:136-46

Lin WW, Karin M. A cytokine-mediated link between innate immunity, inflammation, and cancer. J Clin Invest 2007;117: $1175-83$

Lu LH, Lian YY, He GY, Lin SP, Huan SH, Chen ZZ. Clinical study of triptolide in treatment of acute leukemia. Clin Exp Investig Hematol 1992;3:1-3

Malliri A, van Es S, Huveneers S, Collard JG. The Rac exchange factor Tiam 1 is required for the establishment and maintenance of cadherin-based adhesions. J Biol Chem 2004;279:30092-8

Mudter J, Neurath MF. II-6 signaling in inflammatory bowel disease: pathophysiological role and clinical relevance. Inflamm Bowel Dis 2007;13:1016-23

Onose J, Imai T, Hasumura M, Ueda M, Hirose M. Rapid induction of colorectal tumors in rats initiated with 1,2-dimethylhydrazine followed by dextran sodium sulfate treatment. Cancer Lett 2003;198:145-52

Phillips PA, Dudeja V, McCarroll JA, Borja-Cacho D, Dawra RK, Grizzle WE, Vickers SM, Saluja AK. Triptolide induces pancreatic cancer cell death via inhibition of heat shock protein 70. Cancer Res 2007;67:9407-16

Qiu D, Kao PN. Immunosuppressive and anti-inflammatory mechanisms of triptolide, the principal active diterpenoid from the Chinese medicinal herb Tripterygium wilfordii Hook. f. Drugs R D 2003;4:1-18

Ridley AJ. Rho GTPases and cell migration. J Cell Sci 2001;114:2713-22

Schmidt BM, Ribnicky DM, Lipsky PE, Raskin I. Revisiting the ancient concept of botanical therapeutics. Nat Chem Biol 2007;3:360-6

Shamon LA, Pezzuto JM, Graves JM, Mehta RR, Wangcharoentrakul S, Sangsuwan R, Chaichana S, Tuchinda P, Cleason P, Reutrakul V. Evaluation of the mutagenic, cytotoxic, and antitumor potential of triptolide, a highly oxygenated diterpene isolated from Tripterygium wilfordii. Cancer Lett 1997;112:113-7

Simeone-Penney MC, Severgnini M, Rozo L, Takahashi S, Cochran BH, Simon AR. PDGF-induced human airway smooth muscle cell proliferation requires STAT3 and the small GTPase Rac1. Am J Physiol Lung Cell Mol Physiol 
2008;294:L698-704

Simon AR, Vikis HG, Stewart S, Fanburg BL, Cochran BH, Guan KL. Regulation of STAT3 by direct binding to the Rac1 GTPase. Science 2000;290:144-7

Tengchaisri T, Chawengkirttikul R, Rachaphaew N, Reutrakul V, Sangsuwan R, Sirisinha S. Antitumor activity of triptolide against cholangiocarcinoma growth in vitro and in hamsters. Cancer Lett 1998;133:169-75

Wei X, Gong J, Zhu J, Wang P, Li N, Zhu W, Li J. The suppressive effect of triptolide on chronic colitis and TNF-alpha/TNFR2 signal pathway in interleukin-10 deficient mice. Clin Immunol 2008;129:211-8

Westfall SD, Nilsson EE, Skinner MK. Role of triptolide as an adjunct chemotherapy for ovarian cancer. Chemotherapy 2008;54:67-76

Yang S, Chen J, Guo Z, Xu XM, Wang L, Pei XF, Yang J, Underhill CB, Zhang L. Triptolide inhibits the growth and metastasis of solid tumors. Mol Cancer Ther 2003;2:65-72

Yao J, Jiang Z, Duan W, Huang J, Zhang L, Hu L, He L, Li F, Xiao Y, Shu B, Liu C. Involvement of mitochondrial pathway in triptolide-induced cytotoxicity in human normal liver L-02 cells. Biol Pharm Bull 2008;31:592-7

Zampetaki A, Zhang Z, Hu Y, Xu Q. Biomechanical stress induces IL-6 expression in smooth muscle cells via Ras/Rac1-p38 MAPK-NF-kappaB signaling pathways. Am J Physiol Heart Circ Physiol 2005;288:H2946-54 